\title{
Acción Antimicrobiana in vitro de la Miel de Abejas sobre los Microorganismos Cariogénicos Estreptococos del Grupo mutans
}

\author{
In vitro Antimicrobial Activity of Honey on mutans Streptococci
}

"Luis A. Salazar; "*Felipe Medina; **Francisco Donoso; "Leticia Barrientos \& ${ }^{* * * *}$ Antonio Sanhueza

\begin{abstract}
SALAZAR, L. A.; MEDINA, F.; DONOSO, F.; BARRIENTOS, L. \& SANHUEZA, A. Acción antimicrobiana in vitro de la miel de abejas sobre los microorganismos cariogénicos estreptococos del grupo mutans. Int. J. Morphol., 27(1):77-82, 2009.

RESUMEN: En el presente estudio fue evaluada la actividad antimicrobiana in vitro de cuatro muestras de mieles producidas en nuestro país, sobre los recuentos de estreptococos del grupo mutans, en escolares con alto riesgo de caries dental. Se obtuvieron muestras de saliva de 20 escolares, con edades entre 12 y 14 años, pertenecientes a la ciudad de Temuco, Región de La Araucanía (Chile). El recuento de estreptococos del grupo mutans en saliva fue estimado por el método microbiológico semi-cuantitativo Linoscreen ${ }^{\circledR}$. La evaluación de la acción antibacteriana de la miel se realizó en 9 escolares que presentaron los recuentos más elevados de estreptococos del grupo mutans, utilizando concentraciones de miel entre 5\% y 35\%. Los datos mostraron que el 100\% de los niños analizados poseían colonias de estreptococos del grupo mutans en su saliva. Además, se verificó que el 45\% (9/20) de los escolares se encontraba en la categoría de alta actividad cariogénica. Con este estudio, también se comprobó que la miel de abejas posee actividad antimicrobiana sobre las bacterias estreptococos del grupo mutans y que no existían diferencias significativas entre las 4 mieles utilizadas, con relación a su capacidad antibacteriana ( $>$ >0.05). Por otra parte, este estudio también permitió demostrar que a mayor concentración de miel utilizada mayor era la reducción de las bacterias cariogénicas. En conclusión, en el presente estudio se demostró la acción antimicrobiana in vitro de la miel sobre los recuentos de bacterias cariogénicas estreptococos del grupo mutans. Sin embargo, serán necesarios futuros estudios para identificar y evaluar los componentes de la miel de abejas, responsables de esta propiedad.
\end{abstract}

PALABRAS CLAVE: Caries; Estreptococos del grupo mutans; Actividad antibacteriana; Miel.

\section{INTRODUCCIÓN}

En Chile, la caries dental es considerada uno de los principales problemas de Salud Pública, debido a su alta prevalencia y severidad. Según el Ministerio de Salud (MINSAL), un $84.7 \%$ de la población entre 6 y 8 años presenta evidencias clínicas de caries y un $84.3 \%$ de la población de 12 años (Urbina et al., 1999). Sin embargo, en la población adulta, la prevalencia de caries puede llegar hasta 100\% (Gamonal, 1996).

En esta patología infecciosa, las bacterias estreptococos del grupo mutans se asocian con mayor frecuencia al desarrollo de lesiones cariosas (Ruiz et al., 2007). Estos microorganismos desempeñan un papel preponderante en la patogenia de la caries, debido a su capacidad para favo- recer la desmineralización del diente. Con el objeto de evitar la acción de estas bacterias se han utilizado diferentes estrategias y procedimientos. Es así, como se ha investigado y demostrado la acción antimicrobiana de diversos productos naturales sobre las bacterias productoras de caries (Hayacibara et al., 2005; Massoth et al., 2006; Prabu et al., 2006; Huang et al., 2006; Burt, 2006; Chung et al., 2006; Smullen et al., 2007; Liu et al., 2007), entre ellos, la miel de abejas.

Algunos estudios sugieren que la miel de abejas puede ser de gran utilidad en el tratamiento de diferentes enfermedades periodontales, úlceras bucales y gingivitis (George et al., 1978; Elbagoury \& Rasmy, 1993; Molan, 2001a; English et al., 2004). Además, utilizando mieles no selec-

\footnotetext{
* Laboratorio de Biología Molecular \& Farmacogenética, Depto. de Ciencias Básicas, Facultad de Medicina, Universidad de La Frontera, Temuco, Chile.

** Alumnos Carrera de Odontología, Facultad de Medicina, Universidad de La Frontera, Temuco, Chile.

${ }^{* * * *}$ Departamento de Matemáticas \& Estadísticas, Facultad de Ingeniería, Ciencias y Administración, Universidad de La Frontera, Temuco, Chile.

Este trabajo fue financiado por Proyecto FONDEF de CONICYT No D05I10021 y por la Dirección de Investigación y Desarrollo, Universidad de La Frontera, Temuco, Chile (Proyecto DIDUFRO EP 120335).
} 
cionadas, Basson et al. (1994) determinaron la concentración inhibitoria mínima de miel para 7 especies de Streptococcus relacionadas al desarrollo de caries dental. La concentración inhibitoria mínima de miel para Streptococcus oralis fue $12 \%$, para Streptococcus anginosus $17 \%$, y para Streptococcus gordonii, Streptococcus mutans, Streptococcus salivarius, Streptococcus sanguis y Streptococcus sobrinus fue $25 \%$.

Sin embargo, en un estudio en que se utilizaron mieles con actividad antimicrobiana conocida o seleccionadas, las concentraciones inhibitorias mínimas para Streptococcus sobrinus variaron entre 7.5 - 8.5\% v/v (Molan, 2001a). En otro trabajo, se registró que la concentración inhibitoria mínima de miel para Streptococcus mutans y Streptococcus sobrinus fue $25 \%$ y 35\%, respectivamente (Molan, 2001a). Además, se demostró que el recuento de bacterias en saliva, medido una hora después de mantener $5 \mathrm{~mL}$ de miel en la boca durante 4 minutos, disminuyó un 40\% (Molan, 2001a).

Por su parte, Sela et al. (2000) al evaluar el recuento de Streptococcus mutans en 12 individuos controles y 12 pacientes con cáncer de cabeza y cuello, sometidos a radioterapia, después de mantener en su boca $5 \mathrm{~mL}$ de miel por 5 minutos, observaron una reducción significativa del recuento bacteriano en los pacientes con cáncer, sugiriendo que la miel de abejas puede ser considerada un agente antibacteriano en pacientes con xerostomía inducida por la radioterapia.

En base en las informaciones descritas, el presente trabajo tiene como propósito evaluar la acción antibacteriana in vitro de 4 tipos de miel, producidas en nuestro país, sobre los recuentos de los microorganismos cariogénicos estreptococos del grupo mutans, en escolares con alto riesgo de caries.

\section{MATERIAL Y MÉTODO}

Sujetos. Este estudio fue llevado a cabo en 80 escolares de la ciudad de Temuco (Región de La Araucanía, Chile). Cada niño fue sometido a un examen clínico oral, donde se evaluó la historia de caries de acuerdo a los estándares OMS, a través del índice COPD (dientes cariados, obturados y perdidos por caries). Los resultados fueron registrados en una ficha clínica precodificada.

De los 80 escolares evaluados inicialmente, se seleccionaron para participar en este estudio 20 niños (13 varones y 7 mujeres), con edades entre 12 y 14 años, debido a su mayor número de lesiones cariosas (promedio de 7 dientes cariados).
La participación de los escolares en esta investigación fue autorizada por sus padres y/o apoderados. Es importante destacar también, que este proyecto de investigación fue aprobado por el Comité de Ética de la Facultad de Medicina de la Universidad de La Frontera, Chile.

Obtención de las muestras de saliva. Se realizó la toma de muestra de saliva según el método descrito por Linossier et al. (2003), que tiene como requisitos, que el día de la realización del examen, el paciente asista al laboratorio en ayunas y sin haberse cepillado los dientes esa mañana.

Las muestras de saliva fueron tomadas mediante estimulación de la secreción salival, para lo cual los escolares debieron masticar un trozo de 0,9 gramos de parafina sólida durante 2 minutos. Durante este periodo, se le solicitó a cada escolar que acumulara la saliva en el vestíbulo labial inferior, lo cual permitió sumergir una espátula plástica de 7,5 x 0,8 centímetros; luego la espátula se colocó en el interior de un tubo con medio de cultivo líquido selectivo para el desarrollo de colonias de estreptococos del grupo mutans [caldo TYCSB ( $\mathrm{T}=$ casitone; $\mathrm{Y}=$ extracto de levadura; $\mathrm{C}=$ cistina; $\mathrm{S}=$ sacarosa; $\mathrm{B}=$ bacitracina) y rojo neutro; SOPROMED S.A., Santiago, Chile].

Estudio microbiológico por método semicuantitativo. Después de incubar los tubos por $48 \mathrm{~h}$ a $37^{\circ} \mathrm{C}$ en estufa de cultivo, las espátulas con las colonias adheridas de estreptococos del grupo mutans fueron observadas por transiluminación en una lente de magnificación de Spencer, para luego ser ubicadas por categorías de acuerdo al estándar de clasificación recomendado por el método Linoscreen ${ }^{\circledR}$ (SOPROMED S.A., Santiago, Chile). De esta manera, las muestras se ubicaron dentro de tres rangos de riesgo cariogénico, mediante el siguiente criterio de concentración:

- Bajo riesgo: 10.000 - 50.000 UFC/mL de saliva

- Moderado riesgo: 100.000 - 250.000 UFC/mL de saliva

- Alto riesgo: 500.000 - 1.000.000 UFC/mL de saliva.

\section{Estudio in vitro de la actividad antimicrobiana de la} miel. Para el estudio de la actividad antibacteriana de la miel de abejas, se contactó a los 9 escolares que presentaron los recuentos más elevados de estreptococos del grupo mutans (500.000 a 1.000.000 UFC/mL), a los cuales se les realizó nuevamente el estudio microbiológico, de la misma forma descrita previamente. Además, en esta ocasión se les solicitó a los escolares que depositaran la saliva acumulada en tubos estériles, los cuales fueron correctamente cerrados y la tapa sellada con papel parafilm para evitar intercambio de gases. Estas muestras de saliva fueron utilizadas para el estudio de la acción antimicrobiana de la miel. 
En condiciones estériles, se acondicionaron tubos con $5 \mathrm{~mL}$ de caldo de cultivo TYCSB - rojo neutro y volúmenes crecientes de miel, de forma que se obtuvieron las siguientes concentraciones: $5,15,30$ y $35 \%$ v/v. Las mieles utilizadas en este estudio, provenían de diferentes zonas geográficas de la Región de La Araucanía. Entre los criterios de selección de las mieles se consideró que fueran puras y no elaboradas.

Luego, espátulas plásticas fueron sumergidas en los tubos que contenían la saliva de los escolares, y colocadas en los tubos con diferentes concentraciones de miel. Como control positivo se utilizó, en cada batería, un tubo que contenía solamente medio TYCBS y rojo neutro, al cual también se le introdujo una espátula con saliva. Después de incubar los tubos por $48 \mathrm{~h}$, a $37^{\circ} \mathrm{C}$, se procedió a realizar el conteo de las colonias de estreptococos en cada tubo.

Análisis estadístico. El análisis de los datos obtenidos se realizó utilizando los programas SigmaStat para Windows, versión 2.0 y GraphPad Prism, versión 3.0 (EE.UU). Inicialmente, todas las variables fueron analizadas descriptivamente. Se calcularon promedios, desviaciones standard, valores máximos y mínimos. La asociación entre las diferentes variables analizadas fue verificada mediante el método no paramétrico de Cochran-Mantel-Haenszel. Además, se utilizó el test de Duncan para las comparaciones múltiples. El nivel de significancia estadístico considerado fue $\mathrm{P}<0.05$.

\section{RESULTADOS}

Nivel de infección por estreptococos del grupo mutans. La Tabla I muestra los rangos de riesgo cariogénico, según el recuento de estreptococos del grupo mutans en saliva de 20 escolares. Se verificó que el 45\% (9/20) de los escolares analizados, se encontraban en la categoría de alta actividad cariogénica. Además, se observó que el 100\% de los niños presentaba estreptococos del grupo mutans en su saliva.

Evaluación de actividad antimicrobiana in vitro de la miel. Al analizar los recuentos iniciales de estreptococos del grupo mutans, de los 9 escolares seleccionados para el estudio de la acción antimicrobiana de la miel, no se observaron diferencias significativas entre los recuentos de bacterias obtenidos en días diferentes $(800.000 \pm 273.861$ vs. 750.000 $\pm 288.675 \mathrm{UFC} / \mathrm{mL}, \mathrm{p}=0.798)$.

Se verificó que las cuatro muestras de miel analizadas en este estudio presentaban similar actividad antibacteriana sobre estos microorganismos $(\mathrm{p}=0.7156)$, como puede ser comprobado en la Tabla II. Además, todas las concentraciones de miel investigadas $(5,15,30$ y $35 \%)$ produjeron inhibición del crecimiento de estreptococos del grupo mutans, en diferentes magnitudes ( $<<0.0001)$.

Sin embargo, las concentraciones de miel que ocasionaron una mayor reducción del número de unidades formadoras de colonias de estreptococos del grupo mutans fueron 30 y $35 \%$ v/v (Tabla II), no existiendo diferencias significativas entre estas dos concentraciones, según el método estadístico de comparaciones múltiples de Duncan.

Tabla I. Rangos de riesgo cariogénico, según recuento de estreptococos del grupo mutans en saliva de 20 escolares, estimados mediante el método semicuantitativo Linoscreen ${ }^{\circledR}$.

\begin{tabular}{lcc}
\hline Rango de Riesgo & Recuento (UFC/mL) & $\boldsymbol{n}$ \\
\hline Bajo & $10.000-50.000$ & 3 \\
Mediano & $100.000-250.000$ & 8 \\
Alto & $500.000-1.000 .000$ & 9 \\
\hline
\end{tabular}

UFC, Unidad formadora de colonias; n, número de individuos.

Tabla II. Reducción del número de UFC/mL (Promedio \pm DS) de estreptococos del grupo mutans, según tipo y concentración de miel utilizada.

\begin{tabular}{cccccc}
\hline Miel $^{\text {a b }}$ & Recuento inicial ${ }^{*, c}$ & $\mathbf{5 \%}^{\mathrm{d}}$ & $\mathbf{1 5 \%}^{\mathrm{e}}$ & $\mathbf{3 0 \%}^{\mathrm{f}}$ & $\mathbf{3 5 \%}$ \\
\hline M01 & $750.000 \pm 288.675$ & $562.500 \pm 314.576$ & $275.000 \pm 165.831$ & $225.000 \pm 202.072$ & $202.500 \pm 224.406$ \\
M02 & $750.000 \pm 288.675$ & $437.500 \pm 125.000$ & $312.500 \pm 125.000$ & $162.500 \pm 103.077$ & $162.500 \pm 103.077$ \\
M03 & $750.000 \pm 288.675$ & $625.000 \pm 250.000$ & $437.500 \pm 125.000$ & $275.000 \pm 165.831$ & $200.000 \pm 100.000$ \\
M04 & $750.000 \pm 288.675$ & $562.500 \pm 314.576$ & $337.500 \pm 197.378$ & $137.500 \pm 75.000$ & $102.500 \pm 105.000$ \\
\hline
\end{tabular}

*El recuento inicial corresponde al promedio de UFC/mL de los 9 escolares con alto riesgo de caries del mismo día en que fue realizada la evaluación de la actividad antibacteriana de la miel.

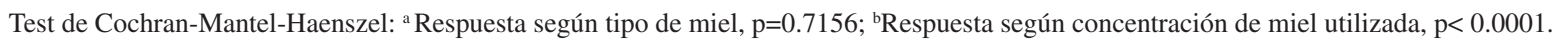

Test de comparaciones múltiples de Duncan: ${ }^{c}$ Recuento inicial vs. diferentes concentraciones de miel, p $<0.05 ;{ }^{\mathrm{d}}$ Concentración $5 \%$ vs. concentración $15 \%$, p $<0.05 ;{ }^{e}$ Concentración $15 \%$ vs. concentración 30\%, p $<0.05 ;{ }^{\mathrm{f}}$ Concentración 30\% vs. concentración $35 \%$, p $>0.05$. 


\section{DISCUSIÓN}

Si la miel de abejas es o no dañina para los dientes, ha sido motivo de discusión durante mucho tiempo. Considerando que la miel tiene un alto contenido de azúcares fermentables, sería de esperar que ésta fuera cariogénica. Sin embargo, varios estudios han demostrado que la miel de abejas posee actividad antibacteriana sobre los microorganismos productores de caries (Basson et al.; Molan, 2001a; Sela et al.).

En el presente estudio, fue evaluada la acción antimicrobiana in vitro de 4 muestras de miel, producidas en nuestro país, sobre las bacterias cariogénicas estreptococos del grupo mutans, en escolares con alto riesgo de caries dental.

Al examinar los recuentos de estreptococos del grupo mutans en saliva, llama la atención la elevada presencia de estos microorganismos. La presencia de éstos fue detectada en el 100\% de los escolares analizados. Presencia similar fue observada en 20 niños de la ciudad de Santiago, con edades entre 6 y 8 años, con un promedio de nueve dientes cariados (Linossier et al.). Estos datos confirman la conocida asociación entre la presencia de Streptococcus mutans en saliva y caries dental.

Además, los datos obtenidos en este estudio corroboraron la acción antimicrobiana in vitro de la miel de abejas sobre los microorganismos estreptococos del grupo mutans, y demostraron también, que no existían diferencias importantes de actividad antibacteriana entre las 4 mieles utilizadas. Por otra parte y como era de esperar, las concentraciones de miel de abejas que mostraron mayor actividad antimicrobiana fueron 30 y $35 \% \mathrm{v} / \mathrm{v}$.

El mecanismo de la acción antimicrobiana de la miel de abejas sobre las bacterias estreptococos del grupo mutans puede ser atribuido a varios factores: actividad osmótica de la miel (Steinberg et al., 1996), concentración de peróxido de hidrógeno en la miel, inhibición de la formación de dextrano (Molan, 2001a), contenido de ácidos orgánicos no aromáticos (Mato et al., 2003), concentración de ácido benzoico y sus derivados, contenido de ácido cinámico y sus derivados, y concentración de flavonoides (Molan, 2001a; Molan, 2001b).

Si bien los datos observados en nuestro estudio, hacen sugerir que la miel de abejas podría ser utilizada como un agente antibacteriano, el elevado consumo de alimentos y bebidas cariogénicas, entre las cuales se encuentra la miel, pueden llevar a un desequilibrio microbiano, favore- ciendo el desarrollo de la caries dental. Bowen \& Lawrence (2005) no recomiendan que los niños consuman miel por causa de los componentes de ésta. Por su parte, Dasanayake \& Caufield (2002) observaron una alta prevalencia de caries en niños aborígenes de Sri Lankan, la cual fue asociada al consumo excesivo de azúcar y miel.

Por otro lado, Molan (2001a) propone que, a pesar de que la miel pueda ser considerada cariogénica debido a su alto contenido de azucares fermentables; si se utilizan mieles que posean una elevada actividad antibacteriana, el potencial efecto sobre el diente puede ser balanceado por la inhibición de las bacterias cariogénicas. Además, se ha demostrado que la miel contiene un compuesto que protege el fosfato de calcio (componente del esmalte dental) de la disolución en ácido; este agente protector, parece ser un éster de fosfato orgánico (Jenkins et al., 1959). En un estudio que evaluó la microdureza del esmalte, mediante microscopía electrónica, no fue observada ninguna evidencia de descalcificación después de la exposición del esmalte dental a diferentes concentraciones de miel $(25,50 \mathrm{y}$ $100 \%$ v/v) durante 3 horas (Grobler et al., 1994).

Estos datos sugieren que la miel a utilizar en un futuro estudio, debe ser previamente caracterizada en cuanto a sus componentes químicos, orígenes botánico y geográfico para obtener mejores resultados. Será necesario conocer también, qué tipos de flores favorecen la producción de mieles con mayor actividad antimicrobiana, y si los resultados obtenidos en este estudio son reproducibles in vivo. Además, es importante destacar que es necesaria la realización de una futura investigación que involucre un mayor número de individuos, de modo de poder validar los hallazgos observados.

SALAZAR, L.A.; MEDINA, F.; DONOSO, F.; BARRIENTOS, L. \& SANHUEZA, A. In vitro antimicrobial activity of honey on mutans streptococci. Int. J. Morphol., 27(1):77-82, 2009.

SUMMARY: The aim of the present issue was to evaluate the antibacterial activity of four commercial honeys against mutans streptococci in schoolchildren with high risk of caries development. We have investigated 20 schoolchildren from Temuco city (Chile), aged $12-14$ years. Saliva samples were obtained by stimulation with solid paraffin. The count of mutans streptococci in saliva was estimated by microbiological method. The antimicrobial activity of honey was evaluated by dilution in TYCBS medium in 9 schoolchildren $(>500.000 \mathrm{cfu} / \mathrm{mL})$. Four concentrations of honey were used ranging from 5 to $35 \%$. The presence of $S$. mutans was detected in $100 \%$ of schoolchildren. Our data shown that honey present high antimicrobial activity against $S$. mutans, being the 
dilutions at 30 and $35 \%$ that shown the better inhibitory effect $(\mathrm{p}<0.001)$. No differences between 4 honeys in relation to antibacterial activity were observed ( $p>0.05)$. In conclusion, our data showed that honeybees present in vitro antibacterial activity against $S$. mutans. However, future studies are necessary to characterize the honey components, responsible for this property.

KEY WORDS: Caries; Streptococcus mutans; antimicrobial activity; Honey.

\section{REFERENCIAS BIBLIOGRÁFICAS}

Basson, N. J.; Du Toit, I. J. \& Grobler, S.R. Antibacterial action of honey on oral Streptococci. J. Dent. Assoc. S. Afr., 49: 339-41, 1994.

Bowen, W. \& Lawrence, R. Comparison of the cariogenicity of cola, honey, cow milk, human milk, and sucrose. Pediatrics., 116(4):921-6, 2005.

Burt, B. A. The use of sorbitol- and xylitol-sweetened chewing gum in caries control. J. Am. Dent. Assoc., 137(2):190-6, 2006.

Chung, J.Y.; Choo, J.H.; Lee, M.H. \& Hwang, J.K. Anticariogenic activity of macelignan isolated from Myristica fragrans (nutmeg) against Streptococcus mutans. Phytomedicine, 13(4):261-6, 2006.

Dasanayake, A. \& Caufield, P. Prevalence of dental caries in Sri Lankan aboriginal Veddha children. Int. Dent. J., 52(6):438-44, 2002.

Elbagoury, E. F. \& Rasmy, S. Antibacterial action of natural honey on anaerobic bacteroides. Egypt. Dent. J., 39(1):381-6, 1993.

English, H. K.; Pack, A. R. \& Molan, P. C. The effects of manuka honey on plaque and gingivitis: A pilot study. $J$. Int. Acad. Periodontol., 6(2):63-7, 2004.

Gamonal, J. Prevalencia de enfermedades periodontales y de caries dental en la población de 35-44 y de 65-74 años de nivel socio-económico bajo y medio-bajo de la provincia de Santiago, Región Metropolitana, y determinación de los recursos humanos necesarios para su tratamiento. Rev. Fac. Odontol. Univ. Chile, 14(1):56-7, 1996.

George, Y.; Sadek, L. \& Roziek, F. The effect of honey on the epithelial attachment. J. Mo. Dent. Assoc., 58 (2):159, 1978.
Grobler, S.R.; Du Toit, I.J. \& Basson N.J. The effect of honey on human tooth enamel in vitro observed by electron microscopy and microhardness measurements. Arch. Oral. Biol., 39 (2):147-53, 1994.

Hayacibara, M.F.; Koo, H.; Rosalen, P.L.; Duarte, S.; Franco, E.M.; Bowen, W.H.; Ikegaki, M. \& Cury, J. A. In vitro and in vivo effects of isolated fractions of Brazilian propolis on caries development. J. Ethnopharmacol., 101(1-3):110-5, 2005

Huang, B. B.; Fan, M.W.; Wang, S. L.; Han, D. X.; Chen, Z. \& Bian, Z. The inhibitory effect of magnolol from Magnolia officinalis on glucosyltransferase. Arch. Oral. Biol., 51(10):899-905, 2006.

Jenkins, G. N.; Forster, M. G. \& Speirs, R.L. The influence of the refinement of carbohydrates on their cariogenicity. Br. Dent. J., 106:362-74, 1959.

Linossier, A.; Pizarro, F.; Potocnjak, P.; Silva, N.; Zillmann, G. \& Larroque C. Frecuencia de biotipos de Estreptococcus mutans en escolares chilenos. Rev. Méd. Chile, 115:411-15, 1987.

Linossier, A.; Vargas, A.; Zillmann, G.; Arriagada, M.; Rojas, R. \& Villegas, R. Streptococci mutans: a semiquantitative method to assess the risk to oral infection in preschool Chilean children. Rev. Med. Chil., 131:412-8, 2003.

Liu, X.T.; Shi, Y.; Yu, B.; Williams, I.D; Sung, H.H.; Zhang, Q.; Liang, J.Y.; Ip, N.Y. \& Min Z.D. Antibacterial diterpenoids from Sagittaria pygmaea. Planta. Med., 73(1):84-90, 2007.

Massoth, D.; Massoth, G.; Massoth, I.R, Laflamme, L.; Shi, W.; Hu, C. \& Gu, F. The effect of xylitol on Streptococcus mutans in children. J. Calif. Dent. Assoc. 34(3):231-4, 2006.

Mato, I.; Huidobro, J.F.; Simal-Lozano, J. \& Sancho, M.T. Significance of nonaromatic acids in honey. J. Food. Prot., 66:2371-6, 2003.

Molan, P.C. The potential of honey to promote oral wellness. General. Dentistry., 49 (6):584-9, 2001 (a).

Molan, P.C. Why honey is effective as a medicine. 2. The scientific explanation of its effects. Bee. World, 82(1):22 40, 2001 (b).

Prabu, G. R.; Gnanamani, A. \& Sadulla, S. Guaijaverin - a 
SALAZAR, L. A.; MEDINA, F.; DONOSO, F.; BARRIENTOS, L. \& SANHUEZA, A. Acción antimicrobiana in vitro de la miel de abejas sobre los microorganismos cariogénicos estreptococos del grupo mutans. Int. J. Morphol., 27(1):77-82, 2009.

plant flavonoid as potential antiplaque agent against Streptococcus mutans. J. Appl. Microbiol., 101(2):48795, 2006.

Ruiz, A.; Montiel, J. M. \& Almerich, J.M. Evaluation of caries risk in a young adult population. Med. Oral. Patol. Oral. Cir. Bucal., 12(5):E412-8, 2007.

Sela, M. Maroz, D. \& Gedalia, I. Streptococcus mutans in saliva of normal subjects and neck and head irradiated cancer subjects after consumption of honey. J. Oral. Rehabil., 27: 269-270, 2000.

Smullen, J.; Koutsou, G.A.; Foster, H.A.; Zumbe, A. \& Storey, D.M. The antibacterial activity of plant extracts containing polyphenols against Streptococcus mutans. Caries Res., 41(5):342-9, 2007.

Steinberg, D.; Kaine, G. \& Gedalia, I. Antibacterial effect of propolis and honey on oral bacteria. Am. J. Dent. 9 (6):236-9, 1996.

Urbina, T.; Caro, J.P. \& Vicent, M. Caries y fluorosis en niños de 6 a 8 años y 12 años. MINSAL, Chile. Departamento de Estadísticas e Información. 1996-1999.
Correspondencia:

Prof. Dr. Luis Antonio Salazar

Departamento de Ciencias Básicas

Facultad de Medicina

Universidad de La Frontera

Av. Francisco Salazar 01145

Casilla 54-D

Temuco, CHILE

Tel.: +56 45592895

Fax: +56 45592831

Email: Isalazar@ufro.cl

Recibido : 09-09-2008

Aceptado: $14-10-2008$ 